\title{
Changes of MK medium during storage of human cornea
}

\author{
SYED M HASANY AND PRASANTA K BASU \\ From the Department of Ophthalmology, University of Toronto, Canada
}

SUMMARY By comparing the composition of McCarey-Kaufman (MK) medium before and after corneal storage we attempted to identify specific physiological changes in the medium as predictors of tissue damage. We also tried to determine if hydrocortisone (a lysosomal membrane stabiliser) added to the medium could reduce tissue damage during storage. Corneas (human and rabbit) were stored in the MK medium with and without hydrocortisone for 4 days at $4^{\circ} \mathrm{C}$. The water and nitrogen contents of the stored cornea were compared with those of the fresh cornea. The medium was analysed before and after corneal storage to determine the concentrations of glucose, protein, and amino acids as well as $\mathrm{pH}$ and osmolarity. Scanning electron microscopy (SEM) was used to estimate the degree of the corneal endothelial cell damage. The nitrogen contents and dry weights of the steroid treated and untreated stored corneas were similar to those of the fresh unstored cornea. The steroid treated cornea contained a lesser amount of water than the untreated cornea. The cornea stored in medium without steroid took up a greater amount of glucose from the medium than the cornea stored in medium with steroid. As compared with their concentrations in the fresh unused medium the concentrations of leucine, lysine, and glycine were lower and that of glutamic acid was higher in both the media used for corneal storage. However, the steroid treated storage medium as compared with the untreated storage medium had a greater reduction in the lowering of leucine, lysine, and glycine, and a lesser reduction in the increase of glutamic acid. Steroid treated medium also had a lesser amount of protein released from the stored cornea. Changes in the $\mathrm{pH}$ and osmolarity of the media before and after corneal storage were not remarkable. SEM showed that the endothelial cells of the cornea stored in the medium containing steroid were less damaged than those of the cornea stored in the medium without steroid.

An alteration of the chemical composition of the aqueous humour of donor eyes stored in the moist chamber can adversely affect the corneal endothelium.' From this we speculated that if the tissue culture medium (McCarey-Kaufman (MK) medium) containing a stored cornea is not replenished, its chemical composition would change by the tissue products and that the change in the composition of the medium might cause adverse effects on the endothelium of the stored cornea. In this experiment we studied the nature of the biochemical alteration of the storage medium. We also tried to see if the addition of steroid ${ }^{2-5}$ in the medium would influence the alteration of the chemistry of the medium. We

Correspondence to Dr P K Basu, Department of Ophthalmology, University of Toronto, 1 Spadina Crescent, Toronto, Ontario M5S 2J5. analysed the storage medium for the absence or reduction (due to the influx or entrance into the stored cornea) and presence or increase (due to the efflux or exit from the stored cornea) of certain specific biochemical substances in the medium. As our previous works ${ }^{2-5}$ showed that steroids can retard corneal autolysis, we studied the effect of hydrocortisone on the entrance and exit of these specific substances into and from the stored tissue.

\section{Methods and materials}

Full-thickness human and rabbit corneas were stored in the MK medium in the presence and absence of hydrocortisone (hydrocortisone 21 sodium succinate, Sigma Chemical, St Louis, Mo) at $4^{\circ} \mathrm{C}$ for 4 days. The medium actually used for corneal storage was called 
the 'used' medium in contrast to the 'unused' medium (fresh medium), which did not at any time come in contact with the tissue. The used medium was of two kinds: (a) experimental medium which contained the steroid, and $(b)$ control medium which did not contain it. The particular storage time was chosen because it was observed previously by Liao et al..$^{5}$ that about this time a moderate amount of cellular damage occurs in the cornea stored in tissue culture media. After this time the compositions of the experimental and the control media were analysed. The fresh medium was also simultaneously analysed. A comparison of the three types of media (experimental, control, and fresh) was then done in terms of their glucose, protein, and amino acid contents as well as their $\mathrm{pH}$ and osmolarity.

All biochemical data were expressed with reference to a given diameter $(11 \mathrm{~mm})$ of the full-thickness cornea or in terms of a given amount of corneal nitrogen.

\section{HUMAN CORNEA}

Eye bank eyes received within 12 hours of death were used. A pair of human donor eyes ( $n=10$ pairs) were sterilised by the usual eye bank procedures, and the corneas with a thin scleral rim (about $1 \mathrm{~mm}$ ) were excised. One of the pair of corneas was placed in the experimental medium $(20 \mathrm{ml})$ containing $10^{-6} \mathrm{M}$ hydrocortisone and the other cornea in the same quantity of the control medium.

\section{RABBIT CORNEA}

As human corneas were not available in a fresh state (i.e., immediately after the donors' death), in order to study the effect of storage on corneas having a minimal post-mortem autolytic change, corneas of rabbits soon after their death (fresh cornea) were used. Healthy albino rabbits (3-4 kg) were killed with an overdose of sodium pentobarbital (Nembutal, Abbott Laboratories, Montreal). Both eyes of a rabbit were immediately enucleated and sterilised by the usual eye bank procedure. The corneas $(n=$ 10 pairs) with a thin scleral rim (about $1 \mathrm{~mm}$ ) were excised and stored in the experimental and control media as done in the case of the human corneas. Additional fresh rabbit corneas $(n=4)$ were also analysed for their water and nitrogen contents.

\section{TECHNIQUES USED ON THE CORNEA}

(a) Measurement of dry weight. Each sample of the cornea with a rim of sclera (corneal sample) was rinsed in cold saline and transferred into a preweighed test tube. The tube was kept in a desiccator at $60^{\circ} \mathrm{C}$ for 18 hours, and then reweighed with a Mettler H18 balance (Fisher Scientific Co., Don
Mills, Ontario). The difference in weights was the dry weight of the corneal sample.

In order to estimate the dry weight of the cornea proper (i.e., without the scleral rim), a number of full-thickness trephined discs, $11 \mathrm{~mm}$ in diameter (corneal disc) were obtained from freshly enucleated rabbit eyes as well as from human donor eyes. The dry weights of the corneal discs were determined by the technique mentioned above.

(b) Determination of total nitrogen. Each of the dried corneal samples and corneal discs were treated with $0.5 \mathrm{ml}$ of a mixture containing sulphuric acid in a saturated solution of copper sulphate. After the completion of digestion the amount of nitrogen in the given amount of tissue was estimated by the microKjeldahl technique. ${ }^{6}$

(c) Scanning electron microscopy (SEM). Corneal samples were fixed in the universal fixative $(1 \%$ glutaraldehyde and $4 \%$ formalin in phosphate buffer, $\mathrm{pH} \mathrm{7.2)} \mathrm{at} 4^{\circ} \mathrm{C}$ for 24 hours. ${ }^{2}$ The tissue was washed several times with phosphate buffer $(\mathrm{pH} 7 \cdot 2)$, and was postfixed in $1 \%$ buffered osmium for one hour at room temperature. The tissue was dehydrated in ethanol and dried by the carbon dioxide critical point method. The cornea was mounted with the endothelial side up on a stub and sputter-coated with gold. The specimen was examined by a scanning electron microscope (S-180-SEM Cambridge, England). From the photomicrographs the ratio of the damaged and undamaged cells was calculated.

\section{TECHNIQUES USED ON THE MEDIA}

(a) Determination of glucose. The glucose concentration of the MK media was determined using Otoluidine reagent (Sigma Technical Bulletin 635, Sigma Chemical Co., St Louis, Mo). Each sample of the medium was centrifuged at $2000 \mathrm{rpm}$ for 5 minutes (Sorvall, GLC-1 Newton, CT). The absorbance of the supernatant was measured at $635 \mathrm{~nm}$ with a spectrophotometer (Beckman DU 2400, Beckman Instrument Inc, Fullerton, CA). Standard glucose solutions were used as reference.

(b) Measurement of protein. The protein content of the MK media was determined by two methods-UV wavelength method Whitaker and Granum ' and Lowry and colleagues' method. ${ }^{\mathrm{B}}$ Bovine serum albumin was used as the reference standard in both instances.

(c) Amino acid analysis. Amino acid concentrations of the media were determined both before and after hydrolysis of the samples. Hydrolysis was done to break down proteins coming out from the stored cornea into amino acids.

With respect to the unhydrolysed sample, $50 \mu \mathrm{l}$ aliquots of the medium were assayed with an amino acid analyser (Beckman model 12/M, Palo Alto, 
Table 1 Analysis of human and rabbit full-thickness corneal discs

\begin{tabular}{|c|c|c|c|c|c|c|c|c|}
\hline & \multicolumn{4}{|l|}{ Human } & \multicolumn{4}{|l|}{ Rabbit } \\
\hline & \multicolumn{2}{|c|}{ Stored in medium } & \multirow{2}{*}{$\begin{array}{l}\text { Unstored } \\
\text { Fresh } \\
\text { (C) }\end{array}$} & \multirow{2}{*}{$\begin{array}{l}\text { Significance } \\
\text { of difference } \\
\text { (p) } \\
(B-A)\end{array}$} & \multicolumn{2}{|c|}{ Stored in medium } & \multirow{2}{*}{$\begin{array}{l}\text { Unstored } \\
\text { Fresh } \\
\text { (C) }\end{array}$} & \multirow{2}{*}{$\begin{array}{l}\text { Significance } \\
\text { of difference } \\
(p) \\
(B-A)\end{array}$} \\
\hline & $\begin{array}{l}\text { With } \\
\text { steroid } \\
(A)\end{array}$ & $\begin{array}{l}\text { Without } \\
\text { steroid } \\
\text { (B) }\end{array}$ & & & $\begin{array}{l}\text { With } \\
\text { steroid } \\
(A)\end{array}$ & $\begin{array}{l}\text { Without } \\
\text { steroid } \\
\text { (B) }\end{array}$ & & \\
\hline $\begin{array}{l}\text { Dry weight } \\
\text { (mg) }\end{array}$ & $24 \cdot 94 \pm 0 \cdot 01$ & $25 \cdot 12 \pm 0 \cdot 04$ & $24 \cdot 94 \pm 1.07$ & $<0 \cdot 2$ & $16 \cdot 70 \pm 0 \cdot 90$ & $18 \cdot 47 \pm 1 \cdot 90$ & $16 \cdot 30 \pm 1 \cdot 38$ & $<0 \cdot 2$ \\
\hline $\begin{array}{l}\text { Nitrogen } \\
\text { content } \\
\text { (mg) }\end{array}$ & $2 \cdot 96 \pm 0 \cdot 17$ & $3 \cdot 06 \pm 0 \cdot 01$ & $2 \cdot 91 \pm 0 \cdot 50$ & $<0.5$ & $2 \cdot 0 \pm 0 \cdot 5$ & $2 \cdot 11 \pm 0 \cdot 10$ & $2 \cdot 10 \pm 0 \cdot 11$ & $<0.5$ \\
\hline $\begin{array}{l}\text { Water } \\
\text { content } \\
(\mathrm{mg})\end{array}$ & $107 \pm 7 \cdot 00$ & $122 \pm 6 \cdot 00$ & $108 \cdot 57 \pm 5 \cdot 20$ & $<0.05$ & $68 \cdot 60 \pm 8 \cdot 00$ & $86 \cdot 80 \pm 2 \cdot 00$ & $62 \cdot 90 \pm 4 \cdot 00$ & $<0.01$ \\
\hline
\end{tabular}

California). With reference to the hydrolysed medium, $1 \mathrm{ml}$ aliquots of the medium were transferred into a hydrolysing tube and lyophilysed overnight. For hydrolysis $0.5 \mathrm{ml}$ of $6 \mathrm{~N} \mathrm{HCl}$ was added into the tube, sealed under vacuum, and heated at $100^{\circ} \mathrm{C}$ overnight. The hydrolysate $(50 \mu \mathrm{l}$ aliquots $)$ was then assayed with the analyser. Appropriate amino acid solutions were used as the reference standards. The amino acid analysis was done by the Amino Acid Analysing Service, Medical Sciences Building, University of Toronto, Toronto, Canada.

(d) Measurement of osmolarity. Standard filter paper discs were saturated with the medium and the osmolarity measured with a vapour pressure osmometer (Model 5100 B Wescor, Inc., Logan, Utah). Standard $\mathrm{mmol} / \mathrm{kg}$ solutions were used as reference.

(e) Measurement of $\mathrm{pH}$. The $\mathrm{pH}$ of the medium was determined with a pH meter (Beckman Zeromatic SS 3, Beckman Instruments Inc., Fullerton, Ca).

\section{STATISTICAL ANALYSIS}

The difference between the amount of a given substance in the fresh medium and the amount of the same substance in the experimental or control medium was determined. The difference in the amount of a target agent in the fresh cornea and the amount of the same substance in the stored cornea was also determined. The significance of the difference of the values in each case was analysed by means of the Student's $t$ test and/or Wilcoxon sign rank test.

\section{Results}

Corneal analysis. As shown in Table 1, in the dry weight and nitrogen content there was no significant difference between the steroid treated and untreated stored corneas, nor was there any difference between either of these and the fresh corneas. However, the water content of the cornea stored in the experimental medium was significantly lower than that of the cornea stored in the control medium. Water content of the fresh cornea was lower than that of the corneas stored in the control medium, but it was similar to that in the experimental cornea.

Media analysis. The results related to the human cornea are given in Figs. 1 and 2, and that related to

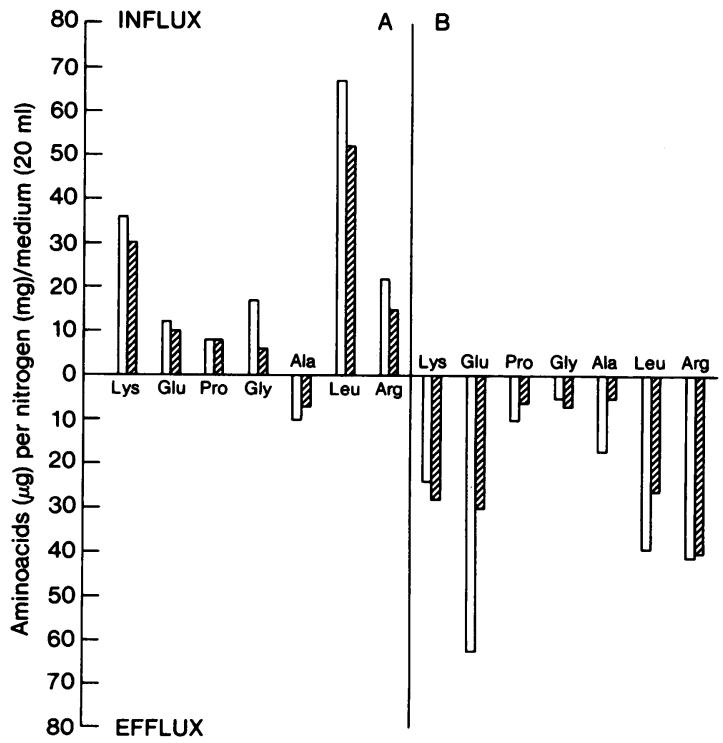

Fig. 1 Amino acids in medium with hydrocortisone (striped bar) and without hydrocortisone (open bar) used to store human cornea, A, unhydrolysed medium, B, hydrolysed medium. The line at the 0 mark represents the values of unstored fresh medium. The values above the line indicate the loss of a given amino acid from the media (i.e., influx into the cornea. The values below the line indicate the gain of a given amino acid into the medium (i.e., efflux from the cornea)). 


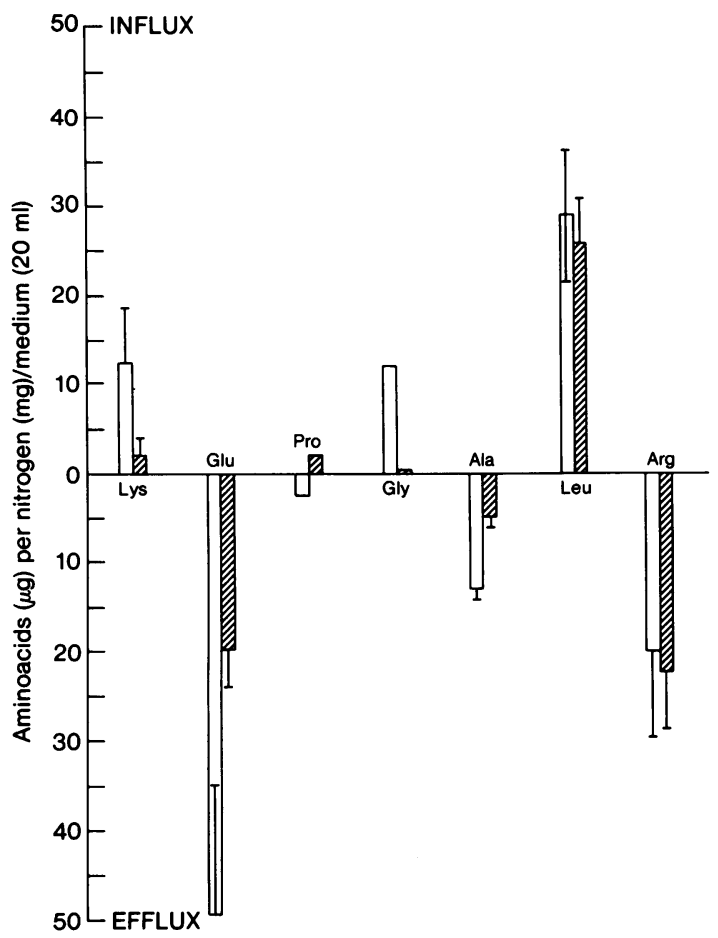

Fig. 2 Net efflux and influx of amino acids in medium with hydrocortisone (striped bar) and without hydrocortisone (open bar) used to store human cornea. Standard error of mean is shown.

the rabbit cornea in Fig. 3. Any increase or decrease of a given substance in the experimental and control medium above or below the quantity of the substance present in the fresh medium has been considered to have resulted respectively from a leaching out of the substance (efflux) from the stored cornea or an entrance of the substance (influx) into the cornea from the medium.

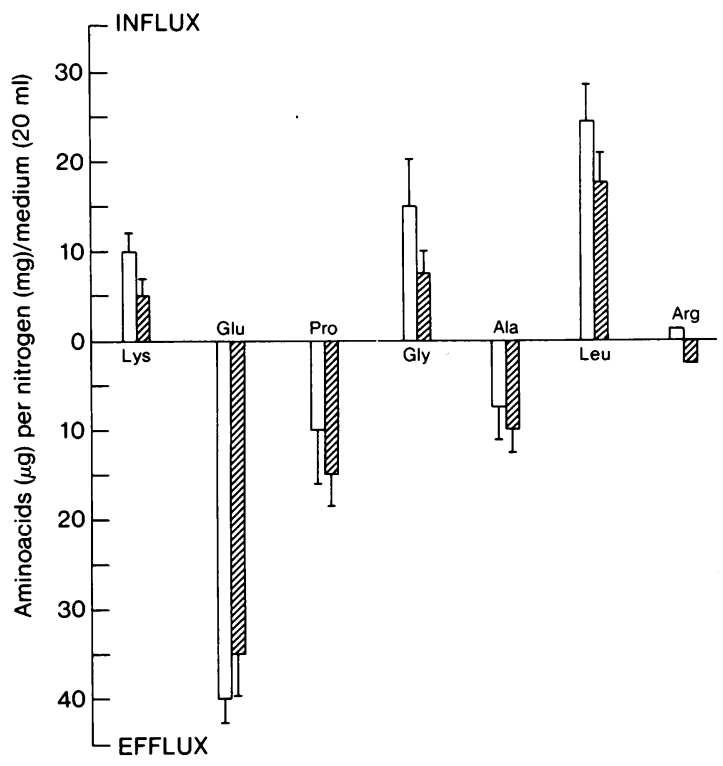

Fig. 3 Net efflux and influx of amino acids in medium with hydrocortisone (striped bar) and without hydrocortisone (open bar) used to store rabbit cornea. Standard error of mean is shown.

Glucose. Table 2 shows that in the control medium there was a greater loss of glucose than in the experimental medium $(\mathrm{p}<0.05)$.

Protein. Both the methods of Whitaker and Granum ${ }^{7}$ and of Lowry et al. ${ }^{8}$ were used to determine the protein concentration and showed that the control medium had a greater quantity of protein than the experimental medium (Table 2).

Amino acids. A comparison of the amino acid contents of the different media showed that certain amino acids were more and others were less in the experimental or control medium than that in the

Table 2 Analysis of MK medium following storage of human and rabbit full-thickness corneal discs, $11 \mathrm{~mm}$ in diameter

\begin{tabular}{|c|c|c|c|c|c|c|}
\hline & \multicolumn{3}{|l|}{ Human cornea } & \multicolumn{3}{|l|}{ Rabbit cornea } \\
\hline & $\begin{array}{l}\text { Medium with } \\
\text { steroid } \\
\text { (A) }\end{array}$ & $\begin{array}{l}\text { Medium without } \\
\text { steroid } \\
\text { (B) }\end{array}$ & $\begin{array}{l}\text { Significance of } \\
\text { difference }(p) \\
(B-A)\end{array}$ & $\begin{array}{l}\text { Medium with } \\
\text { steroid } \\
\text { (A) }\end{array}$ & $\begin{array}{l}\text { Medium without } \\
\text { steroid } \\
\text { (B) }\end{array}$ & $\begin{array}{l}\text { Significance } \\
\text { of difference } \\
(p) \\
(B-A)\end{array}$ \\
\hline Glucose $\mu \mathrm{g} / \mathrm{ml} / \mathrm{disc}$ & $22 \pm 10$ & $124 \pm 40$ & $<0.05$ & $88 \pm 20$ & $114 \pm 20$ & $<0.05$ \\
\hline $\begin{array}{l}\text { Protein (Lowry) } \\
\mu \mathrm{g} / \mathrm{ml} / \text { disc }\end{array}$ & $22 \cdot 80 \pm 6 \cdot 3$ & $29 \cdot 78 \pm 5 \cdot 6$ & $<0.01$ & $20 \cdot 58 \pm 3 \cdot 7$ & $30 \cdot 60 \pm 3 \cdot 3$ & $<0.05$ \\
\hline $\begin{array}{l}\text { Protein (UV) } \\
\mu \mathrm{g} / \mathrm{ml} / \mathrm{disc}\end{array}$ & $42 \cdot 28 \pm 14 \cdot 2$ & $56 \cdot 20 \pm 20 \cdot 0$ & $>0.05$ & $38 \cdot 60 \pm 3 \cdot 6$ & $54 \cdot 44 \pm 7 \cdot 2$ & $<0.05$ \\
\hline $\mathrm{pH}^{*}$ & $8 \cdot 2 \pm 0 \cdot 1$ & $8 \cdot 31 \pm 0 \cdot 1$ & $<0 \cdot 10$ & $8 \cdot 30 \pm 0 \cdot 1$ & $8 \cdot 34 \pm 0 \cdot 1$ & $<0 \cdot 20$ \\
\hline $\begin{array}{c}\text { Osmolarity }{ }^{\dagger} \\
\mathrm{mmol} / \mathrm{kg}\end{array}$ & $331 \pm 5 \cdot 0$ & $338 \pm 7 \cdot 0$ & $<0 \cdot 10$ & $325 \pm 3 \cdot 0$ & $330 \pm 1 \cdot 0$ & $<0.02$ \\
\hline
\end{tabular}

${ }^{*} \mathrm{pH}$ of fresh medium was $7 \cdot 5 \pm 0 \cdot 2$.

†Osmolarity of fresh MK medium was $320 \pm 2 \mathrm{mmol} / \mathrm{kg}$. 
fresh medium as represented by the line at 0 in the Figs. 1 and 2. Hydrolysis of the medium manifested a greater amount of a given amino acid as compared with its corresponding quantity in unhydrolysed medium (Fig. 1). The net quantity of a given amino acid in the storage medium (control and experimental) was the difference in the values obtained from the hydrolysed and unhydrolysed media (Figs. $2,3)$.

Fig. 1 shows the amino acid data for the unhydrolysed and hydrolysed samples of the experimental and control media related to the human cornea. In the unhydrolysed sample the concentrations of leucine and lysine were lower $(<20 \mu \mathrm{g} / \mathrm{ml})$ (Fig. 1A) and in the hydrolysed sample the concentration of glutamic acid was higher $(>20 \mu \mathrm{g} / \mathrm{ml})$ than their respective concentrations in the fresh medium (Fig. 1B).

As shown in Fig. 2, the net quantity of a given amino acid in the medium in which the human cornea was stored (the experimental or control medium) was different from that of the same amino acid in the fresh medium. As compared with the fresh medium, both storage media had $(a)$ a greater amount of glutamic acid, alanine, and arginine and $(b)$ a lesser amount of leucine, lysine, and glycine. However, the exit from and the entrance into the cornea of most of these amino acids were both influenced in the presence of the steroid. When the steroid was present in the medium, we saw a reduction in the increase of glutamic acid and alanine on the one hand, and a reduction in the decrease of leucine, lysine, and glycine on the other hand (Fig. 2).

Comparing Fig. 3 with Fig. 2 we see that the rabbit cornea also showed a trend similar to that shown by the human cornea. As in the case of human cornea, hydrocortisone decreased the quantity of leucine and lysine in the medium, and also reduced the increase of glutamic acid into the media.

Scanning electron microscopy (SEM) was done on three paired corneas of rabbit-one cornea being stored in the experimental medium and the other in the control medium. The cornea kept in the control medium showed a higher percentage of damaged endothelial cells (Fig. 4A) as compared with the cornea stored in the experimental medium $(31 \%$ vs $8 \cdot 2 \%$ ) (Fig. 4B). When these results were compared with that related to the unstored fresh cornea, the latter hardly showed any damaged endothelial cells (Fig. 4C).

There was no significant difference in the $\mathrm{pH}$ between the experimental and control media (Table 2 ). The osmolarity of the experimental medium tended to be slightly lower than that of the control medium, but the difference was not statistically significant (Table 2).
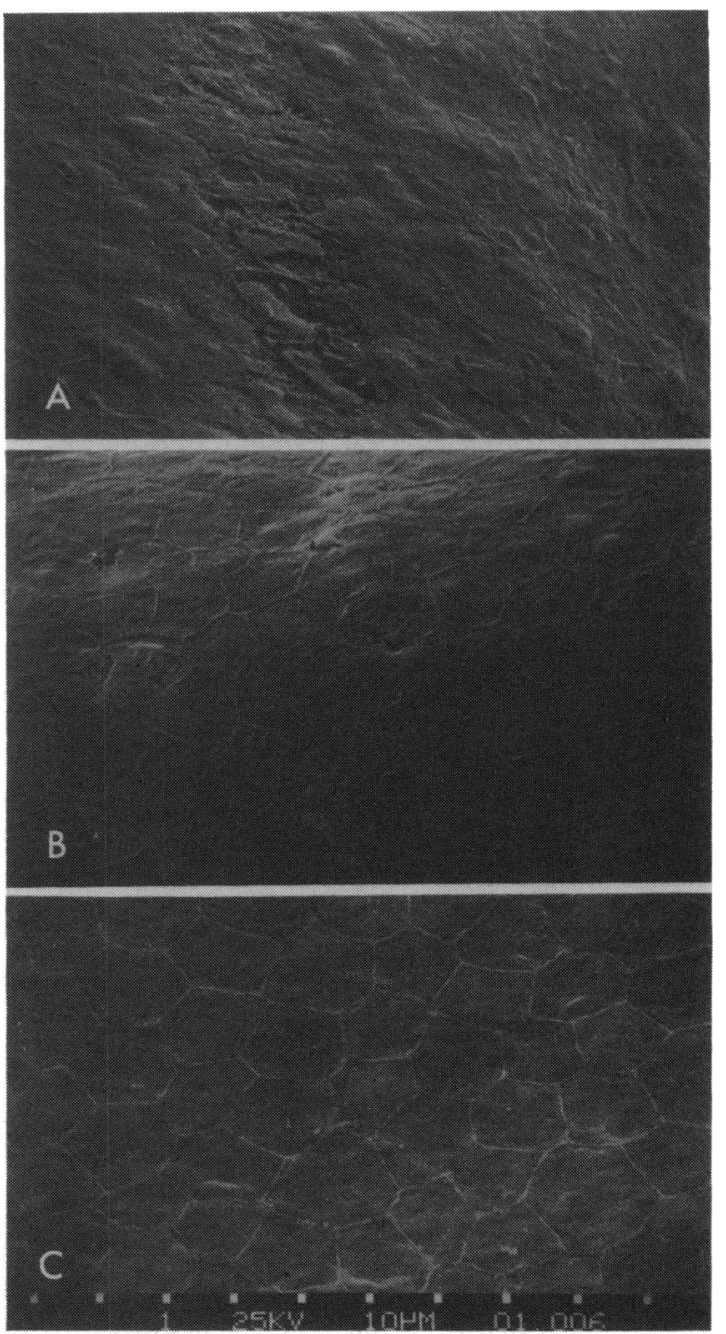

Fig. 4 Scanning electronmicroscopy of the corneal endothelium. A: Cornea stored in the absence of hydrocortisone showing extensive areas of cellular damage. B: Cornea stored in the presence of hydrocortisone showing some damaged cells. C: Fresh unstored cornea showing undamaged cells.

\section{Discussion}

It has been shown that the corneal endothelial cells from the time of the death of the eye donor undergo rapid structural and functional changes which make them more permeable to organic and inorganic substances. ${ }^{9}$ It has also been shown that hydrocortisone (a lysomal membrane stabiliser) by protecting the cell membranes can reduce corneal damage during storage.$^{+5}$ Our present study indicates that the steroid added to the storage medium can influence 
the entrance into and release of certain specific substances from the cornea.

It is interesting to see that in terms of the water content the cornea stored in the MK medium containing hydrocortisone was more similar to the unstored fresh cornea (compare the $p$ values in Table 1) than to the cornea stored in the medium without the steroid. As the dry weights and the nitrogen contents of the corneas stored in the experimental and control media were identical, the greater weight of the cornea stored in the steroid-free MK medium was obviously due to a greater increase in the entrance of water from the medium into the tissue. Hydrocortisone thus reduced the flow of water into the cornea during storage.

Glucose is required by the donor cornea as early as one hour after the enucleation of the eye. ${ }^{10}$ Glucose is one of the major components of the MK medium. Our results showed that both the human and rabbit corneas kept in the steroid-containing medium utilised approximately five times less quantity of glucose than identical corneas stored in medium free of steroid (Table 2). Our glucose results are in agreement with that of Landau," who reported a decrease in the utilisation of glucose by the muscle, adipose tissue, skin, lymphoid tissue, or leucocytes when treated with steroid. Norton and Munck ${ }^{12}$ also found a dose related decrease in glucose uptake by macrophage treated with glucocorticoids in vitro.

Our study showed that a greater amount of protein was present in the control MK medium than in the experimental medium. Hydrocortisone thus reduced the release of protein from the stored cornea into the storage medium.

Although we analysed 20 amino acids in our study, we focused our attention particularly on seven of them, known to be abundant in the protein residue of collagen, ${ }^{13}$ which is an important constituent of the cornea. In comparison with their respective concentrations in the fresh medium, in both the experimental and control media the concentration of leucine was lower and that of alanine and glutamic acid were higher. Although we do not know the mechanism of the amino acid transfer, the influx of leucine and the efflux of alanine and glutamic acid to and from the stored cornea may be associated with cell membrane damage (Fig. 1). A comparison of the experimental and control media suggests the possibilty of reduction of corneal damage (in terms of amino acids) of stored corneas by steroid (Figs. 2 and $3)$. An analysis of the net difference between the influx and efflux values of the different amino acids shows that the entrance of leucine into the cornea and release of glutamic acid from the cornea were quite remarkable (Figs. 2 and 3). As steroid tended to reduce both the entry and exit of these two amino acids to and from the stored cornea, we feel that a study of these amino acids could possibly be utilised in judging the quality of a stored cornea in tissue culture medium.

The human donor eyes, from which the corneas were obtained, varied in terms of the period between death and enucleation, cause of death, donor age, etc. In contrast, the eyes of rabbits were obtained immediately after their death. In view of our previous work $^{2}$ we would assume that when we received the human eyes they had a greater degree of corneal autolysis as compared with the rabbit eyes. It was, therefore, interesting to note that the fresh rabbit corneas and the stored human corneas behaved in a similar fashion with respect to the qualitative pattern of exit from or entrance into the corneas of the amino acids mentioned before. Quantitatively, however, both the experimental and control media related to the rabbit corneas had lower concentrations of leucine and lysine as compared with the experimental and control media related to the human cornea. We believe that the above quantitative difference between the rabbit and human corneas, apart from the species difference, was due to the difference in the degree of autolysis present in the two types of corneas when we started the experiments. However, at present we cannot explain why some of the amino acids were affected in one way and the others in another way.

With regard to the uptake of leucine by the cornea from the storage media, the work of Hanna ${ }^{14}$ seems to be interesting. He reported that corneal cells can absorb leucine and incorporate it into new protein. Scott and Friedenthal ${ }^{15}$ showed that leucine uptake is stimulated by lactate. Since lactate is known to be produced during mid-term storage of the cornea,${ }^{16}$ it is possible that to form new proteins leucine is utilised by the surviving cells in the autolysing stored cornea. In one of our studies (unpublished data) we have seen a gradual increase in the uptake of radioactive leucine from the MK medium (without steroid) into the cornea with the progress of the storage time. In our present study we have seen that hydrocortisone reduced the entrance of leucine from the medium to the cornea. This may be due to a reduced demand for the production of new proteins ${ }^{14}$ in a more favourably stored cornea as a result of the reduction of tissue autolysis by steroid.

The entrance and exit of the amino acids into and from the stored cornea were probably related to the damage of the corneal cell barriers. A comparison of the results of our present SEM study on the corneal endothelium of fresh unstored rabbit eyes with the results of our previous SEM study on the corneal endothelium of stored human eyes ${ }^{2}$ showed that the amount of cell damage in the stored human cornea 
was greater than that in the fresh rabbit cornea. Both our present (Fig. 4) and previous studies ${ }^{2}$ also showed that steroid can reduce structural damage of the corneal endothelial cells from both species.

We did not detect any significant difference in the $\mathrm{pH}$ values among experimental, control, and fresh media. Hull et al. ${ }^{17}$ also did not find any significant alteration of $\mathrm{pH}$ in the medium in which corneas were stored up to seven days, but they found some change after 21 days.

Otori ${ }^{18}$ showed that the electrolyte content of the cornea decreased when the whole eye was stored in the moist chamber. Hull et al. ${ }^{17}$ found a decrease in the potassium content of the cornea stored in the MK medium for seven days. The decrease of the electrolyte content of the stored cornea would tend to increase the electrolyte concentration of the storage medium and thus could be a guide for determining the degree of tissue damage..$^{19}$ In our present study we were unable to detect any statistically significant change of osmolarity of the storage media. However, as shown in Table 2, we noticed a trend towards the reduction of the electrolyte concentration $(\mathrm{mmol})$ of the storage medium when steroid was added to it.

In conclusion, we think that the use of steroid in the storage medium should be beneficial in preventing corneal damage during mid-term and possibly long-term storage of the donor cornea. Further, we believe that a chronological biochemical analysis of the MK medium particularly for the increase of protein and glutamic acid and the decrease of leucine and glucose could be helpful in monitoring the nature and rate of damage to the cornea stored in tissue culture media.

This work was supported by grants from the Medical Research Council of Canada, the Canadian National Institute for the Blind out of the E A Baker Foundation for the Prevention of Blindness, Independent Order of Odd Fellows and Rebekahs of Ontario, and the Lions International District A-16 Ophthalmological Research Foundation, Canada.

\section{References}

1 Basu PK, Hasany SM, Ranadive NS, Chipman ML. Damage to the corneal endothelial cells by lysosomal enzymes in stored human eyes. Can J Ophthalmol 1980; 15: 137-40.
2 Basu PK, Hasany SM, Doane FW, Schultes K. Can steroid reduce endothelial damage in stored corneas? Effect on cell viability and ultrastructure. Can J Ophthalmol 1978; 13: 31-8.

3 Spencer J, Dixon W, Ranadive NS, Basu PK. Factors in the survival of stored corneas. Can J Ophthalmol 1977; 12: 123-7.

4 Liao HR, Hasany SM, Lin BJ, Basu PK. Biochemical analysis of the cornea stored in steroid medium. Can J Ophthalmol 1979; 14: 274-80.

5 Liao HR, Hasany SM, Lin BJ, Basu PK. Effect of steroid on the glucose metabolism in the stored cornea. Metab Pediatr Syst Ophthalmol 1980; 4: 67-70.

6 Elvidge JA, Sammes PG. A course in modern technique of organic chemistry. 2nd ed. London: Butterworth, 1966: 262.

7 Whitaker JR, Granum PE. An absolute method for protein determination based on difference in absorbance at 235 and 280 nm. Anal Biochem 1980; 109: 156-9.

8 Lowry OH, Roseborough NJ, Farr AL, Randall RJ. Protein measurement with the folin phenol reagent. J Biol Chem 1951; 193: 265 .

9 Hoefle FB, Maurice DM, Sibley RC. Methods of evaluating corneal donor material. In: Capella JA, Edelhauser HF, Van Horn DL, eds. Corneal preservation. Springfield: Thomas, 1973: 96-107.

10 Thoft RA, Friend J, Freeman H, Dohlman CH. Corneal epithelial preservation. Arch Ophthalmol 1979; 93: 357-61.

11 Landau BR. Adrenal steroids and carbohydrate metabolism. Vitam Horm 1965; 23: 1-59.

12 Norton JM, Munck A. In vitro action of glucocorticoids on murine macrophages: effects on glucose transport and metabolism, growth in culture and protein synthesis. J Immunol 1980; 125: 259-66.

13 Eastoe JG. Composition of collagen and allied proteins. In: Ramachandran GN, ed. Treatise on collagen. London: Academic Press, 1967: 27-32.

14 Hanna C. Ultrastructural changes and RNA and protein synthesis in cells of human eye bank cornea. In: Capella JA, Edelhauser HF, Van Horn DL, eds. Corneal preservation. Springfield: Thomas, 1973: 39-53.

15 Scott WN, Friedenthal DF. A proposed role for ascorbate in the transport of amino acids and ions in the cornea. Exp Eye Res 1973; 15: 683-92.

16 Schmidt-Martens FW, Hennighausen U, Wirzkrand Teping C. Alterations in the metabolism of corneal epithelium during medium term storage. Graefes Arch Clin Exp Ophthalmol 1977; 203: $145-52$.

17 Hull DS, Green K, Bowman K, Csukas S, Riley MV. Intracellular $\mathrm{pH}$ and glutathione levels in rabbit corneal endothelium following storage in moist chamber and MK medium. Invest Ophthalmol Vis Sci 1983; 24: 214-7.

18 Otori T. Electrolyte content of the rabbit corneal stroma. Exp Eye Res 1967; 6: 356-67.

19 Greiner JV, Lass JH, Glonek T. Ex vivo metabolic analysis of eye bank corneas using phosphorous nuclear magnetic resonance. Arch Ophthalmol 1983; 102: 1171-3.

Accepted for publication 11 August 1986. 